\title{
Médiévales
}

Langues, Textes, Histoire

62 | printemps 2012

Hagiographie et réforme dans l'Occident latin

\section{Célébrer l'église, réformer la personne : la fête de la dédicace d'église dans La Légende dorée de Jacques de Voragine}

Celebrating the Church, Reforming the Individual. The Feast of the Dedication of a Church in the Golden Legend by Jacobus de Voragine

Dominique logna-Prat

\section{OpenEdition}

Journals

Édition électronique

URL : https://journals.openedition.org/medievales/6677

DOI : 10.4000/medievales.6677

ISSN : $1777-5892$

Éditeur

Presses universitaires de Vincennes

Édition imprimée

Date de publication : 2 juin 2012

Pagination : 123-133

ISBN : 978-2-84292-346-4

ISSN : 0751-2708

Référence électronique

Dominique logna-Prat, « Célébrer l'église, réformer la personne : la fête de la dédicace d'église dans La Légende dorée de Jacques de Voragine », Médiévales [En ligne], 62 I printemps 2012, mis en ligne le 28 juin 2012, consulté le 22 avril 2022. URL : http://journals.openedition.org/medievales/6677 ; DOI : https://doi.org/10.4000/medievales.6677 
Médiévales 62, printemps 2012, p. 123-134

Dominique IOGNA-PRAT

\section{CÉLÉBRER L'ÉGLISE, RÉFORMER LA PERSONNE : LA FÊTE DE LA DÉDICACE D'ÉGLISE DANS LA LÉGENDE DORÉE DE JACQUES DE VORAGINE}

La présente étude est née du désir d'aborder le phénomène de la sainteté paradoxale ou, pour le moins, de cas limites du sanctoral dans l'hagiographie latine médiévale. On connaît nombre de saints plus ou moins «bricolés » (souvent plus que moins), dont les coordonnées hagiographiques jouent de références dans le temps et dans l'espace largement inventées. Telle est la rançon d'une tradition qui repose sur la culture des restes de morts considérés comme «très spéciaux» au bénéfice d' «impresarii » obéissant aux nécessités présentes ${ }^{1}$. Le problème a été à ce point ressenti, dès le Moyen Âge, qu'on a pu célébrer, par dérision, le culte de «saint personne» (sanctus Nemo), personnification absurde du «rien», du néant primordial dramatisé dans le récit de la Genèse ${ }^{2}$.

La sainteté dont il va être question ici est «paradoxale» dans la mesure où la tradition chrétienne a fait d'un «objet» (une construction de pierre) un véritable «sujet». D'où le titre : en célébrant une église «anthropisée», c'est la personne du fidèle qu'il s'agit de «réformer». Le terme «réforme», au centre de la réflexion collective proposée dans ce numéro de Médiévales, est difficile à expliciter du fait de la diversité de ses champs d'application, étant entendu qu'avant la «Réforme» (avec une majuscule), temps d'un tournant radical dans l'histoire du christianisme, on ne parle que de la ou des réforme(s) de

1. P. Brown, Le Culte des saints. Son essor et sa fonction dans la chrétienté latine, trad. fr., Paris, 1984 [éd. orig. Chicago, 1980].

2. Nemo (ficticius): BHL 6050v-z; cf. M. BAYLess, Parody in the Middle Ages: the latin Tradition, Ann Arbor, 1996. Ce traitement dérisoire de la personne du «rien» touche pour partie à la question de l'incroyance au Moyen Âge: D. WelteCKE, «Der Narr spricht : es ist kein Gott». Atheismus, Unglauben und Glaubenszweifel vom 12. Jahrhundert bis zur Neuzeit, Francfort - New York, 2010. 
l'Église, de telle communauté, de la liturgie, et ainsi de suite ad libitum, au gré des besoins d'une «Église mixte» (comme disait Augustin), ni radicalement mauvaise, ni vraiment bonne, sujette à de nécessaires et incessants ajustements . Entre mille références possibles, je choisis de partir de la définition fournie par le sociologue des religions François-André Isembert: "[la réforme] est le dynamisme par lequel se développe, au sein d'un système religieux, le besoin d'expulser comme inauthentique une part des manières de faire et de penser autrefois reçues ${ }^{3} . »$ Le rejet de l'«inauthentique» par le saint serait le point focal du «système religieux»; dans le «système chrétien», il se situerait au point d'articulation entre communauté et personne du fidèle. Le saint, porteur des valeurs de la communauté ecclésiale, personnifierait en quelque sorte la «réforme», entendue comme nécessaire restauration de la personne. En bref, j'aimerais poser le problème de la «sainte réforme», de «la réforme par les saints » dans la logique du personnalisme chrétien, lequel veut que la personne et la loi privée prévalent toujours sur les potentielles errances de la communautét ${ }^{4}$.

Le dossier qui va nous permettre d'examiner cette question de la réforme ecclésiale par le médium de la personne est fourni par le dernier chapitre de la seconde version de La Légende dorée de Jacques de Voragine ${ }^{5}$. Dans un ensemble thématique consacré au haut Moyen Âge, il s'agit certes d'un dossier tardif, mais le côté récapitulatif de toute l'histoire des topiques hagiographiques qu'organisent les grands légendiers d'ordres depuis le $\mathrm{XII}^{\mathrm{e}}$ siècle peut offrir un point de vue intéressant pour l'étude du thème proposé, «Réforme(s) et hagiographie». $L a$ Légende dorée est compilée entre 1261 et 1267, mais Jacques de Voragine révise le texte jusqu'à la fin de sa vie (1298). Dans la seconde version, il choisit d'ajouter un chapitre absent de la première rédaction et qui est placé comme une sorte «d'épilogue ecclésiologique» à l'ensemble de l'œuvre: la fête de dédicace d'église ${ }^{6}$. C'est comme si Jacques, dans un ultime repentir, cherchait à récapituler, à rassembler tant de gestes singulières, dans le cadre monumental nécessaire aux effusions communautaires: l'église. Dans l'histoire des légendiers latins, l'initiative de Jacques est sans précédent. On ne trouve rien de tel dans l'abrégé de Jean de Mailly (1225-1230, révisé en 1243), et tout juste une mention, mais

3. F.-A. ISEMBERT, Rite et efficacité symbolique, Paris, 1979, cité par J.-P. WiLlaIme, « Réforme/ réformation», dans R. AzRIA et D. HERVIEU-LÉGER éd., Dictionnaire des faits religieux, Paris, 2010, p. 1004-1012 (p. 1005).

4. P. von Moos, «L'individu ou les limites de l'institution ecclésiale », dans B. BEDos-ReZAK et D. Iogna-Prat éd., L'Individu au Moyen Âge. Individuation et individualisation avant la modernité, Paris, 2005, p. 271-288.

5. On suivra ici l'édition critique fournie par G. P. MAGGioni, Florence, 1999, et la traduction publiée sous la direction d'A. Boureau, Paris, 2004 (Bibliothèque de la Pléiade), désormais citées respectivement Legenda aurea et La Légende dorée.

6. La Légende dorée, p. 1041-1057. 
pas finale, dans le Liber epilogorum in gesta sanctorum de Barthélemy de Trente (1245). La logique à l'œuvre dans ce choix fait dans un second temps reste encore à expliquer, les études de référence n'ayant pas jusqu'à maintenant abordé la question, à la notable exception d'un article de Florent Coste ${ }^{7}$.

Pour saisir au mieux cette logique, il convient de resituer le propos de Jacques dans un cadre large. La célébration de la fête de dédicace fait d'abord sens dans l'ensemble de La Légende dorée, qui comporte de nombreux récits de construction d'églises, tandis que les hasards du sanctoral amènent le compilateur à articuler le thème de la sainte conversion à celui d'une construction, à évoquer la lente émergence d'une parure monumentale chrétienne avec la destruction des temples païens et la construction d'églises ou la conversion de temples en églises, ou mieux encore à traiter des colonnes de l'Église que sont les saints - à commencer par Dominique (avant François d'Assise), soutien de l'Église vacillante dans le fameux songe d'Innocent III $^{8}$. Un pareil intérêt pastoral pour la construction édifiante est largement confirmé par les sermons de Jacques, à commencer par ses sermons de dédicace: on construit pour instruire, l'église étant considérée comme l'espace de la parole ${ }^{9}$. Enfin, il convient de rappeler, à la suite des travaux de Cécile Caby, l'importance accordée par les dominicains, malgré la mobilité structurelle de l'ordre, à la consécration de leur domus et de ses lieux, à commencer par le dortoir, comme s'il fallait lutter pièce après pièce contre le diable et comme si l'irradiation du locus specialis initialement consacré, l'église, ne suffisait pas ${ }^{10}$. Dernier élément de contextualisation du chapitre de La Légende dorée relatif à la fête de dédicace de l'église: la concomitance de l'initiative de Jacques avec l'attention manifestée par deux exégètes de la liturgie contemporains: Guillaume d'Auxerre (v. 1218-1228), dans sa Summa de officiis ecclesiasticis, et Guillaume Durand (v. 1230-1296), dans le Rational des divins offices, lesquels choisissent aussi de placer l'église au sein du sanctoral ${ }^{11}$. Voyons maintenant, à l'examen du chapitre de Jacques de Voragine, quelle impérieuse nécessité il y avait à intégrer l'église-bâtiment dans la communio sanctorum.

7. F. Coste, «Construction et édification de l'ecclesia selon Jacques de Voragine», dans C. Dauphant et V. Obry éd., Rêves de pierre et de bois. Imaginer la construction au Moyen Âge, Paris, 2009, p. 49-71.

8. Legenda aurea $109, \S 57$, p. 722 ; La Légende dorée, p. 586; pour François, voir la Légende de Thomas de Celano et, bien sûr, la fresque de Giotto, à l'église supérieure d'Assise.

9. F. Coste, «Construction et édification...», p. 62-63.

10. C. CABY, «Construction et sacralisation des espaces conventuels dans l'ordre des frères prêcheurs (XIII -début XIV ${ }^{\mathrm{e}}$ siècle», dans Lieux sacrés et espace ecclésial (IX ${ }^{e}$-XV siècle), Cahiers de Fanjeaux, t. 46, Toulouse, 2011, p. 131-171.

11. Gulllaume D’Auxerre, Summa de ecclesiasticis officiis, III, ms Douai, BM 65, fo 77 vb-78 ra; Gulllaume Durand, Rational des divins offices, VII, 48. 
Placée à l'extrême fin du sanctoral, la célébration de la date anniversaire de la dédicace d'église est une fête locale, attachée à la figure d'un saint patron du lieu ou d'un saint universel, mais indépendamment du dies natalis de ce saint. La seule universalité que «canonise » en quelque sorte Jacques de Voragine est le principe général de cette fête anniversaire. Il note en prologue que cette fête est «double»-double au sens liturgique, mais surtout au sens symbolique, puisque «double est l'église ou le temple», à la fois matériel et spirituel. C'est sur cette «duplicité» que la notice est construite.

Le texte traite d'abord du temple matériel en trois questions: pourquoi et comment est-il consacré? Peut-il être profané ? La logique du développement est empruntée à l'exégèse liturgique, à laquelle Jacques se réfère explicitement dans le cas du Mitrale de Sicard de Crémone. Il s'agit pour lui, comme pour l'exégète, d'expliciter le sens, essentiellement moral et allégorique, du rite de dédicace.

\section{La dédicace du temple et de l'autel}

«Église ou temple» (ecclesia siue templum) : l'incertitude de l'appellation mérite d'être soulignée d'emblée, d'autant que Jacques ouvre chaque notice de son légendier par une brève notation étymologique sur le nom du saint. Dans le cas de la «sainte église», c'est sur la confusion église/Église qu'il ne manque pas de jouer. Dans le vocabulaire latin désignant l'église-bâtiment, on a pu noter qu'après un long temps de flottement dans les appellations, le terme ecclesia s'est progressivement imposé au cours du haut Moyen Âge comme terminus technicus, mais sans jamais atteindre l'exclusivité dans la désignation du «lieu». Ce terme est adopté pour son ambiguïté métonymique, «église/Église» renvoyant à la fois au contenant et au contenu ${ }^{12}$. Jacques joue d'ailleurs pleinement de l'équivoque quand il note, à l'entame du texte, que «la dédicace de l'église est célébrée par l'Église».

L'examen de la question commence par la dédicace de l'autel. Ce choix obéit à la logique polarisante implicite au rituel de consécration : c'est par l'autel que l'église/Église rayonne alentour et c'est à l'autel que viennent les fidèles en quête d'incorporation au corps du Christ. Trois raisons expliquent cette dédicace. La première est, bien sûr, d'offrir le sacrement du Seigneur, Jacques notant trois types de commémoration de la Passion, par l'image, le verbe et le sacrement. Il s'agit ensuite d'invoquer le nom du Seigneur, dans le lieu de sa présence, à l'instar d'Abraham (Gen. 12, 7, 8). Enfin, l'autel est un appel au chant et aux douces mélodies, comme l'enseigne la geste de David, le souverain constructeur du Temple de Jérusalem (Sir. 47, 10-11).

12. D. Iogna-Prat, La Maison Dieu. Une histoire monumentale de l'Église (800-1200), Paris, 2006, p. $48 s q$. 
Cinq raisons justifient la consécration du temple ou de l'église. Le rite a pour première fonction de chasser le diable du lieu exclusif du Seigneur. Jacques évoque la puissance purificatrice de la liturgie de dédicace en se référant à un épisode célèbre des Dialogues de Grégoire le Grand (III, 30): le miracle survenu à Saint-Agathe-des-Goths (Rome) à l'occasion de la réaffectation au culte catholique par reconsécration, en 591 ou 592, de l'ancienne église des Goths ariens. Cet épisode offre, sur le terrain hagiographique, l'une des attestations les plus précoces de l'histoire du cérémonial de dédicace dans l'Occident latin, avec la mention de trois éléments constitutifs : déposition des reliques (de saint Sébastien et sainte Agathe), sacrifice eucharistique et, bien sûr, l'exorcisme évoqué par le miracle lui-même, qui met en scène la fuite d'un porc incarnant le diable. Le deuxième objet du rite est d'instaurer un lieu d'asile - allusion à la fonction de refuge et à l'immunité traditionnellement affectée au lieu de culte. La troisième est d'en faire un lieu de prière. La quatrième est de louer Dieu dans les sept heures canoniales, lesquelles sont des heures «spéciales» permettant de commémorer tel temps christique, par exemple matines, qui est l'heure où le Christ est né, a été arrêté, raillé, a dépeuplé l'Enfer, et qui est aussi l'heure attendue du Jugement. Le cinquième et dernier objet est de loin le plus important. Le rite de dédicace permet d'administrer les sacrements de l'Église. L'église-bâtiment, explique Jacques, est conçue comme «l'auberge de Dieu» où sont contenus et distribués les sacrements - les sept sacrements, énumérés ici sous une forme digne d'attention, en les personnalisant, en mentionnant systématiquement leurs destinataires, par exemple «ceux qui y entrent» pour le baptême, ou «ceux qui y demeurent» pour le sacrement de l'ordre, comme s'il importait à la fois de souligner que le lieu de l'effectuation sacramentelle est le creuset de la transformation des personnes évoquées sous l'indéterminé «ceux qui», et de rappeler que c'est aussi l'espace fonctionnel des transformateurs, les clercs.

Jacques passe ensuite au «comment». De la consécration de l'autel, il retient six gestes. Les quatre croix faites aux quatre coins signifient la quadruple charité ou les quatre parties du monde à l'horizon de l'universalisme chrétien. Le circuit autour de l'autel en sept tours évoque les sept vertus d'humilité du Christ, ou bien ses sept cheminements du Ciel au Ciel, via le ventre maternel, la mangeoire, le monde, le gibet, le tombeau et les limbes. Le commentateur retient quatre autres éléments ou objets du rituel - l'aspersion de l'eau, l'encens brûlé, l'onction de chrême, les nappes blanches recouvrant l'autel identifiées à la pureté de l'officiant -, mais il oublie curieusement de mentionner le dépôt des reliques dans l'autel. Comment expliquer ce silence? Parce que l'autel est le lieu exclusif de l'Eucharistie, et que les saints, comme les âmes des martyrs sous l'autel évoquées par l'Apocalypse $(6,9)$, ne sauraient qu'y avoir une place subordonnée? À une époque où la piété eucharistique impose l'omniprésence 
de la «réalité» du sacrifice christique, où l'hagiographie multiplie les récits de miracles attestant cette «réalité », et où le sanctoral lui-même s'enrichit, avec la fête du Corpus Christi, d'une célébration annuelle de l'avènement de Dieu sous une forme humaine, une pareille exclusivité eucharistique de l'autel ne saurait surprendre ${ }^{13}$.

La logique rayonnante de la sacralité chrétienne fait passer de l'autel à l'ensemble de l'église. Le triple circuit que le consécrateur fait autour du bâtiment est assimilé au trajet du Christ du Ciel au Ciel en passant par le monde et les limbes. Il évoque aussi la Trinité, ainsi que les trois ordres de mérite, du moins au plus nombreux et du plus au moins sacré, auxquels est assignée une place spécifique au sein de l'espace ecclésial : les vierges, assimilés au sanctuaire; les continents, placés dans le chœur; les époux, qui se confondent avec le «corps de l'église», appellation usuelle de la nef.

Les trois coups donnés par le consécrateur sur la porte du bâtiment pour manifester et combattre toute présence diabolique signifient le triple droit du Christ sur l'Église au nom de la Création, de la Rédemption et de la promesse de glorification, ou la triple puissance de Dieu au Ciel, dans le monde, en Enfer. L'aspersion d'eau bénite à l'intérieur et à l'extérieur doit permettre de chasser les démons dans l'exorcisme, d'écarter toute malédiction et de bénir le bâtiment. Jacques met une belle insistance à commenter le fait que l'église elle-même, comme toute chose terrestre, a été corrompue et salie par le péché; elle doit donc «expier», ce que l'on ne saurait attendre que d'une personne animée: la «pénitence» de l'église est un premier jalon essentiel de l'anthropisation du bâtiment.

Suivant une à une les étapes du rite de consécration à l'intérieur de l'église, Jacques s'intéresse ensuite aux fondements et à l'élévation de l'Église. Le pavement permet d'évoquer les «fondements» de la communauté. L'inscription de l'alphabet sur le sol du bâtiment renvoie, comme il est classique depuis la première exégèse du rite de dédicace $a u I^{\mathrm{e}}$ siècle, à l'union des deux peuples à travers les deux Testaments, mais la disposition des lettres en croix de saint André révèle l'inversion fondatrice de l'histoire chrétienne, pas seulement entre la droite et la gauche, mais surtout entre la tête et la queue, les premiers de jadis (les juifs) étant désormais placés à la suite des derniers venus (les chrétiens). Les croix peintes sur les murs par le consécrateur permettent de passer du sol fondateur au volume par lequel l'Église gagne en hauteur. Ces croix ont pour fonction de terrifier les démons, de proclamer le triomphe du Christ, et de représenter les douze apôtres, les douze luminaires posés devant les croix signifiant le pouvoir des apôtres à «illuminer le monde». 
Jacques n'en dit pas plus sur le rite de consécration de l'église. Il omet de parler de la messe qui clôt la cérémonie et qui lui donne tout son sens puisque c'est la commémoration du sacrifice du Christ qui engendre la communauté des chrétiens et lui donne «corps». Mais était-il besoin d'insister sur cet aboutissement manifeste pour les contemporains de Jacques, à une époque où le bâtiment est saturé de références à la présence réelle, constante, manifeste de Dieu en son lieu? En manière d'appendice à cette séquence sur le pourquoi et le comment de la dédicace d'église, Jacques aborde la question de l'inversion rituelle, des malheurs de l'église-bâtiment, de sa dégradation et de son avilissement. Depuis le $\mathrm{XII}^{\mathrm{e}}$ siècle, les canonistes ont développé toute une casuistique des cas de profanation du lieu de culte et de la nécessaire réconciliation du bâtiment. $L a$ Légende dorée réserve d'ailleurs une place de choix à ce problème au chapitre 11, consacré à Thomas de Cantorbéry, «ce saint [qui] a subi le martyre pour l'Église, dans une église, en un lieu sacré, en un temps sacré, entre les mains des prêtres et des religieux, afin qu'apparaisse bien la sainteté du martyr et la cruauté des personnes ${ }^{14} \gg$. Par qui le temple peut-il être profané ? Jacques répond à la question sur le mode de l'exemplum, sous la forme du commentaire moral propre à éclairer les déviances qui souillent la pureté de l'Église. Il cite trois types de profanateurs - Jéroboam, Naburzadam et Antiochus -, auxquels répondent trois antitypes Moïse, Salomon et Judas Maccabée. Ce faisant, il s'agit de dénoncer les vices qui polluent l'Église: Jéroboam et les clercs cupides ; Naburzadam, le prince des cuisiniers, emblématique de tous les gourmands et luxurieux qui transforment Dieu en ventre, le temple en cuisine, l'autel en table, les ministres en cuisiniers; Antiochus, figure des orgueilleux, des clercs en quête de domination, que Bernard de Clairvaux dénonce dans l'un de ses sermons sur le Cantique des Cantiques ${ }^{15}$. L'église est l'espace fonctionnel des clercs dont les vices peuvent compromettre la pureté. Maîtres du sacrifice de l'autel, ils doivent dans leur propre oblation ouvrir la voie du sacrifice personnel attendu des fidèles.

\section{La dédicace du temple spirituel}

Traitées sur le mode de l'exemplum, ces figures déviantes ménagent une bonne transition avec la fin de la notice relative à la dédicace du temple spirituel. Le thème est un grand classique dans l'histoire de l'édification chrétienne. Nous sommes le Temple en tant que «pierres vivantes» habitées par l'Esprit, surédifiées sur la «pierre angulaire » qu'est le Christ (I Pr. 2,5) - des pierres polies par l'action cléricale, dont la prédication, des pierres «carrées » symbolisées par la quadrature des vertus théologales (foi, espérance, charité) couronnées par les

14. La Légende dorée, p. 85.

15. Bernard de Clairvaux, Sermo super Canticum, 33, 15. 
œuvres. Tout juste évoqué, le problème apparait ici avec l'évidence d'un pur lieu commun. Pour autant, dans les années 1220, la question du temple spirituel rapportée au temple matériel est encore loin d'être évidente. Riche d'une tradition aussi ancienne que les Pères, la conception immatérielle de l'Église innerve toute une série de courants dissidents, depuis les hérétiques d'Arras (1025) jusqu'aux cathares contemporains de Jacques, attachés à la référence apostolique d'une communauté de personnes habitée de la présence divine dans la logique de la parole de Jésus: "Que deux ou trois soient réunis en mon Nom, je suis là au milieu d'eux» (Matth. 18, 20). Depuis deux bons siècles, les clercs s'emploient à justifier en doctrine ce qui n'est rien d'autre qu'une nécessité négligeable pour les premiers théologiens chrétiens : la présence tangible du divin et la fabrication sacramentelle de la communauté des fidèles dans l'enveloppe matérielle où Dieu vient prendre corps ${ }^{16}$. Nul écho ici des débats sur le terrain de la théologie sacramentelle permettant de justifier cette pétrification monumentale de l'Église. Mais la notice, structurée sur la correspondance des deux temples (matériel et spirituel), est nourrie de tout un terreau exégétique propre à enseigner les «sens» de l'Église: historique dans sa matérialité renvoyant aux édifices de l'Ancien Testament (Temple ou Tabernacle); allégorique, comme anticipation de la Jérusalem céleste; tropologique, comme horizon de l'intériorité de la personne chrétienne, que le propos de Jacques aborde dans le traitement de la persona de l'église/Église. C'est dans ce même jeu sur le contenant et le contenu que les exégètes de la liturgie, tel Guillaume Durand, christianisent l'analogie vitruvienne entre corps humain et édifice, et donnent à l'église-bâtiment une forma humaine, le chœur (la tête), le transept (la poitrine et les bras), et la nef (le buste et les jambes) étant placés dans un pur rapport d'inversion ${ }^{17}$.

La logique de la synecdoque qui gouverne le raisonnement analogique des exégètes amène Jacques à traiter de la partie (l'autel, le cœur) pour l'ensemble (l'église, le corps). Et tout comme il a commencé son évocation de la dédicace du Temple matériel en son centre, il entame celle du Temple spirituel par l'autel du cœur et l'évocation du «sacrifice» personnel. Il y a, bien sûr, correspondance de registre entre sacrifice personnel et sacrifice du Christ, mais on ne répétera jamais assez à quel point, depuis la Réforme grégorienne, ce sacrifice est médiatisé par la persona sacerdotale, l'officiant étant censé entrer «dans l'intimité de son cœur» au moment du canon de la messe ${ }^{18}$. C'est ainsi la voie de l'exemplarité

16. D. Iogna-Prat, La Maison Dieu..., passim.

17. Gullaume Durand, Rational des divins offices, I, i, 14. Sur les destinées médiévales de l'analogie vitruvienne, voir É. DE BRUYNe, Études d'esthétique médiévale, Paris, 1998 [1 $1^{\mathrm{re}}$ éd. Bruges, 1946], t. 1, p. 258-259.

18. Lothaire de Segni, De missarum mysteriis, PL, t. 217, col. 839; Guillaume Durand, Rational des divins offices, IV, 35, 3. Sur cette question, voir A. RAUwEL, «Le lieu cultuel dans la 
sacerdotale qui est offerte au fidèle en quête d'élévation. En ce sens, l'église de la fête de dédicace est d'abord sainte en tant qu'espace fonctionnel des clercs qui y sacrifient à l'autel, «dans l'intimité de [leur] cœur», ouvrant la voie à la démultiplication des cœurs identifiés à l'autel d'une matrice sacrificielle commune. Dans ces conditions, on conçoit que la personne du fidèle soit un temple spirituel consacré de la même façon que le temple matériel, comme l'enseignent d'ailleurs traditionnellement les sermons de dédicace d'église, à commencer par la série de sermons de Bernard de Clairvaux, qui développent de façon systématique toutes les ressources de l'interprétation tropologique pour rappeler non seulement que la véritable consécration est celle des «pierres animées », mais qu'il faut que "la maison ait été consacrée pour que l'âme soit sanctifiée ${ }^{19}{ }$. D'où la série d'équivalences proposées par Jacques: le triple circuit devant la porte fermée du cœur; la triple aspersion et l'effusion des larmes intérieures et extérieures - une expression lacrymale remarquable en contexte ecclésiologique ${ }^{20}$; l'alphabet spirituel qui, selon la parole de l'apôtre, permet d'inscrire la Loi dans son cœur ( $R m$ 2,14-15); les douze croix peintes qui supposent de se charger de dures pénitences en suivant la voie apostolique.

\section{La personnalisation de la monumentale Église}

Dans le passage d'un sens à l'autre, par saut du matériel au registre moral, il ne s'agit pas pour Jacques de Voragine de simplement jouer sur les équivalences ou les correspondances terme à terme entre bâtiment et fidèle, ou entre autel et cœur. La dernière notice de La Légende dorée, dans sa seconde version, obéit à une logique de causalité: pas de temple spirituel sans temple matériel. L' «épilogue ecclésiologique» proposé consiste en une réunion finale de tous les saints dans l'Église signifiée par l'église, sainte personne parce qu'enveloppe nécessaire aux saints contenus dans l'Église. D'où la «personnalisation» qui, si l'on peut dire, «anime» tout le propos de Jacques: l'église fêtée à sa date anniversaire comme un véritable saint; la correspondance établie entre temple matériel et temple spirituel qui permet d'inscrire la personne dans un cadre sacramentel constitutif; le traitement sacramentel de la personne-église, dont Jacques justifie la nécessaire «expiation», dans l'exorcisme, comme création

synthèse liturgique de Guillaume Durand», dans Lieux sacrés et espace ecclésial..., p. 115-130 (p. 127).

19. Bernard de Clairvaux, Sermones in dedicatione ecclesiae, dans Sancti Bernardi Opera, t. V, J. LelercQ et H. Rochais éd., Rome, 1968, I, 4, p. 370-398 (p. 372).

20. Sur «les constructions sans pleurs» de l'ecclésiologie, voir P. NAGY, Le Don des larmes au Moyen Âge, Paris, 2000, p. 329 sq. 
corrompue par la faute; la persona des clercs, maître des rituels, qui font l'Église dans l'église.

Ce faisant, Jacques joue sur le lien ancien qui unit le saint au lieu. Dans la tradition chrétienne, c'est en effet la présence du saint et de ses restes qui fait la sainteté/sacralité du lieu, avant même le développement d'un rituel de consécration, comme l'atteste la double acception de locus à haute époque: reliquaire et lieu de culte. D'ailleurs, La Légende dorée, comme tout légendier, obéit à la logique des «coordonnées hagiographiques» d'abord mise en forme dans les martyrologes: un nom, une date, un lieu. Mais la notice consacrée à la fête de dédicace d'église va bien au-delà de cette logique en sanctifiant le lieu lui-même. On peut voir dans cette inscription de l'église-bâtiment au sanctoral le fruit lointain d'une évolution ecclésiologique dont il convient de rappeler, pour finir, le double mouvement initié à l'âge de la Réforme de l'Église, aux $\mathrm{XI}^{\mathrm{e}}-\mathrm{XII}^{\mathrm{e}}$ siècles : la «monumentalisation» de l'Ecclesia et sa «personnalisation». Le premier mouvement est perceptible dans les nombreuses représentations architecturées de l'ancienne allégorie féminine de l'Ecclesia mater, qui vient proprement épouser la forme du monument communautaire, l'enveloppant à la manière de la Vierge qui donne corps au Christ, ou l'habitant en son «cœur», à l'autel, dans le rôle fonctionnel du sacrificateur ${ }^{21}$.

Comme on l'a rappelé en commentaire de la notice de Jacques, la personnalisation de l'Ecclesia repose d'abord sur une logique langagière: le pouvoir fondateur de la métonymie qui impose un rapport d'équivalence entre église-bâtiment et Église-communauté - un rapport d'équivalence d'abord considéré comme problématique voire abusif, puis justifié en doctrine par le fait que l'église signifie l'Église. C'est dans cette logique que la théologie sacramentelle a pu développer, au courant du $\mathrm{XII}^{\mathrm{e}}$ siècle, une conception de la «causalité instrumentale » propre à justifier la fonction de l'église-vase, enveloppe nécessaire à l'effectuation sacramentelle. Suivant cette conception, la dédicace d'église est assimilée à un «baptême», avec tout un jeu de correspondances entre baptême du monument et baptême de la personne, le premier conçu comme l'indispensable préambule à la réalisation du second comme de l'ensemble de la chaîne sacramentelle qui s'organise alors en septenaire. On comprend ainsi que les exégètes de la liturgie, tel Sicard de Crémone dont s'inspire Jacques de Voragine, abordent la matière de leurs traités systématiques par le lieu avant de s'attaquer à ce qui s'y fait, comme s'il s'agissait là d'un préalable: du contenu par la grâce du contenant. Dans cette évolution remarquable de la conception des médiations chrétiennes (médiations sacramentelles, médiations iconiques, médiations monumentales), la notice finale de la seconde version de La Légende 
dorée marque, avec l'évidence des topiques hagiographiques qui parlent d'ellesmêmes, une manière d'aboutissement. La consécration/dédicace de l'église mérite d'être fêtée comme le temps anniversaire de l'engendrement de la communauté chrétienne, à la fois dans l'anonymat d'une célébration universelle (la fête de dédicace d'église en général) et la particularité d'une histoire locale (la fête de la dédicace de telle église). Notation importante: contrairement aux autres saints, célébrés à la date anniversaire de leur mort, le dies natalis de la «sainte église » est celui de son «baptême», de son avènement à la vie sacramentelle pour le Dieu fait homme et les membres de son corps. Cette célébration baptismale a la logique d'un engendrement; la «sainte personne» de l'église fabrique en son sein d'autres saintes personnes - la multitude des fidèles, «pierres vivantes » dont l'édification ne saurait s'envisager que dans le cadre d'une vaste construction monumentale leur donnant incessamment forme : l’Église.

Dominique Iogna-Prat - École des hautes études en sciences sociales, CEIFR, 10, rue Monsieur le Prince, F-75006 Paris

Célébrer l'église, réformer la personne: la fête de la dédicace d'église dans La Légende dorée de Jacques de Voragine

La sainteté dont il est question dans cette étude est largement paradoxale dans la mesure où la tradition chrétienne a fait d'un objet (l'église, honorée comme un saint à l'occasion de l'anniversaire de la fête de dédicace) un véritable sujet. En célébrant, comme le fait Jacques de Voragine dans la Légende dorée, une église «anthropisée», c'est la personne même du fidèle qu'il s'agit de réformer.

Dédicace - église - Jacques de Voragine - personne - réforme.

Celebrating the Church, Reforming the Individual. The Feast of the Dedication of a Church in the Golden Legend by Jacobus de Voragine

The sort of sanctity that this study addresses is paradoxical in the way that the Christian tradition transforms an object (a church, honoured as a saint on the occasion of the anniversary of the feast of its dedication) into a genuine individuality. As can be seen with Jacobus de Voragine celebrating an «anthropomorphized» church in his Golden Legend, the reform implied by the dedication concerns the church's faithful himself.

Church - consecration of the church - Jacobus de Voragine - reform - individual. 
